\title{
ERRATA
}

\section{Erratum: The $(a \gamma \gamma \gamma)$ vertex and three-photon axion decay in external magnetic fields [JETP 89, 13-17 (July 1999)]}

\section{V. Skobelev}

Moscow State Industrial University, 109280 Moscow, Russia

Zh. Éksp. Teor. Fiz. 116, 2212 (December 1999)

[S1063-7761(99)02612-8]

This article contains the following important misprints:

1. The second formula in Eq. (11) on p. 14 should read $\left(\tilde{\gamma}^{5}\right)^{2}=1$.

2. The last term in Eq. (12a) on p. 14 should be $\tilde{g}^{\alpha \rho} \widetilde{g}^{\beta \sigma}$.

3. The last term in the second formula of Eq. (12d) on p. 14 should be $\varepsilon^{\alpha \beta} \widetilde{g}^{\rho \sigma}$.

4. In expression (13) on p. 15 the denominator should appear raised to the power $1 / 2$.

5. In Eq. (14) on p. $15 e$ in the numerator of the fraction should be replaced with $e^{3}$.

6. The eleventh and twelfth lines of Eq. (17) on p. 16 should read

$$
\begin{aligned}
& \left.+(q \varepsilon \kappa) \varepsilon_{\alpha \alpha^{\prime}}\left(k^{\prime \prime} \varepsilon\right)_{\alpha^{\prime \prime}}\right]-\frac{1}{\left(m^{2}-\tilde{m}^{2}\right)^{3}}\left[( q \varepsilon \kappa ) \left(\kappa_{\alpha}^{(1)} \kappa_{\alpha^{\prime}}^{(2)} \kappa_{\alpha^{\prime \prime}}^{(3)}\right.\right. \\
& \left.+\kappa_{\alpha}^{(1)}\left(\kappa^{(2)} \varepsilon\right)_{\alpha^{\prime}}\left(\kappa^{3} \varepsilon\right)_{\alpha^{\prime \prime}}+\left(\kappa^{(1)} \varepsilon\right)_{\alpha} \kappa_{\alpha^{\prime}}^{(2)}\left(\kappa^{(3)} \varepsilon\right)_{\alpha^{\prime \prime}}+\left(\kappa^{(1)} \varepsilon\right)_{\alpha}\left(\kappa^{(2)} \varepsilon\right)_{\alpha^{\prime}} \kappa_{\alpha^{\prime \prime}}^{(3)}\right)-
\end{aligned}
$$

7. In (17a) on p. 16 in the second term of the expression for $\tilde{m}^{2}$ the first factor should be $\left(q-k^{\prime \prime}\right)^{2}$. 